\title{
THE ASYMPTOTIC AND INTEGRAL CLOSURE OPERATIONS IN MULTIPLICATIVE LATTICE MODULES
}

\author{
SYLVIA M. FOSTER AND JOHNNY A. JOHNSON
}

\begin{abstract}
This paper is primarily concerned with the integral and asymptotic closure operations on a multiplicative lattice relative to the greatest element of a lattice module having the ascending chain condition. We show that a cancellation law holds for the asymptotic closure of elements of the multiplicative lattice and we ultimately show, by means of multiplicative filtrations and filtration transforms, that the asymptotic closure of an element in a multiplicative lattice relative to the greatest element of a lattice module, coincides with its integral closure relative to this element in the lattice module.
\end{abstract}

\section{Introduction}

Investigations by Dilworth [1] have indicated that many properties of ideal theory in Noetherian rings can be carried over to Noether lattices. In particular, Lediaev [4] generalized the asymptotic closure and the integral closure of ideals in Noetherian rings to multiplicative lattices having the ascending chain condition. Sharp, Tiras, and Yassi [10] introduced the concept of the integral closure of an ideal relative to a Noetherian module and S. Foster and J. Johnson [2] established that the asymptotic closure of a ideal, relative to a Noetherian module, coincides with its integral closure relative to this Noetherian module.

In this paper, we give a lattice theoretic characterization of the notion of asymptotic closure and integral closure relative to a module. These new closure operations encompass those developed by Lediaev [4], and Sharp, Tiras, and Yassi [10].

In [9] P. Samuel used the asymptotic properties of high powers of ideals to establish a cancellation law in Noetherian rings. Here, we likewise obtain a cancellation law using the notion of asymptotic closure.

Ultimately, by means of E.W.Johnson's a-transfrom [3] and Taylor's [11] multiplicative filtration and filtration transform, we discover that where $M$ is a principal element of a lattice module satisfying the ascending chain condition, and where $0: M=0$, the asymptotic closure of an element of $\mathcal{L}$, a multiplicative lattice, relative to $M$ coincides with its integral closure relative to $M$. Since not all Noether lattices are lattices of ideals

Received July 27, 2004; revised March 7, 2005.

2000 Mathematics Subject Classification. 06F10, 06F05.

Key words and phrases. Noether lattice, lattice module, multiplicative lattice. 
of a Noetherian ring, Theorem 4 extends M. Nagata's result that the asymptotic and integral closure operations coincide in Noetherian rings [6].

First, we review some basic definitions and terminology. By a multiplicative lattice $\mathcal{L}$ we mean a complete lattice on which there is a commutative, associative, and arbitrary join distributive multiplication. The greatest element (denoted as $I$ ) of the multiplicative lattice is the multiplicative identity. The least element of a multiplicative lattice will be denoted by 0 .

\section{The Asymptotic Closure Relative to a Module}

In this section $\mathcal{L}$ denotes a multiplicative lattice, and $I$ denotes its greatest element. We will use $\mathcal{M}$ to represent a lattice module that satisfies the ascending chain condition. We shall now develop the concept of the asymptotic closure of elements of a multiplicative lattice relative to the greatest element $M$ of an $\mathcal{L}$-module. Lowercase letters will represent elements of $\mathcal{L}$ and uppercase letters will represent elements of $\mathcal{M}$. $\mathbb{R}$ denotes the extended real numbers.

To establish the asymptotic closure operation, we first need to define a pseudovaluation on $\mathcal{L}$. By a pseudovaluation on $\mathcal{L}$, we mean a mapping $v: \mathcal{L} \rightarrow \mathbb{R}$ that satisfies the following:

(P1) $v(0)=\infty$,

(P2) $v(I)=0$,

(P3) for all $a, c \in \mathcal{L}, v(a c) \geq v(a)+v(c)$,

(P4) for all $a, c \in \mathcal{L}, v(a \vee c) \geq \min \{v(a), v(c)\}$.

A pseudovaluation $v$ is said to be homogeneous if for all elements $a$ in $\mathcal{L}$ and for all positive integers $n$, we have $v\left(a^{n}\right)=n v(a)$.

We now define a new pseudovaluation on $\mathcal{L}$ relative to an element of $\mathcal{M}$. Let $M$ be the greatest element of $\mathcal{M}$ and let $b$ be an element of $\mathcal{L}$ such that $b M \neq M$. Then we define a function ${ }_{M} v_{b}: \mathcal{L} \rightarrow \mathbb{R}$ by

$$
M v_{b}(a)=\left\{\begin{array}{l}
\infty, \text { in case } a M \leq b^{n} M \text { for every nonnegative integer } n, \\
n, \text { in case } a M \leq b^{n} M, \text { but } a M \not \leq b^{n+1} M,
\end{array}\right.
$$

where $b^{0}$ is defined to be $I$. It can readily be shown that ${ }_{M} v_{b}$ is a pseudovaluation on $\mathcal{L}$.

Using the function defined above, we can now construct a homogeneous pseudovaluation on $\mathcal{L}$. Let $\mathcal{M}$ be an $\mathcal{L}$-module. Let $M$ be the greatest element in $\mathcal{M}$ and let $b$ be an element of $\mathcal{L}$ such that $b M \neq M$. Then, for the pseudovaluation ${ }_{M} v_{b}$, one can show that for every element $a$ in $\mathcal{L}$, the limit of the sequence $\left\{\frac{M v_{b}\left(a^{n}\right)}{n}\right\}_{n=1}^{\infty}$ exists. The proof of this fact is similar to the proof given on [8, Lemma 1.2, p.109]. We therefore define ${ }_{M} \bar{v}_{b}(a)=\lim _{n \rightarrow \infty} \frac{M v_{b}\left(a^{n}\right)}{n}$ for all $a$ in $\mathcal{L}$. 
By applying properties of multiplicative lattices and the definition of ${ }_{M} v_{b}$, one can show that $M \bar{v}_{b}$ is a homogeneous pseudovaluation on $\mathcal{L}$. Where $b M \neq M$, we set $\hat{b}=$ $\left\{a \in \mathcal{L}:{ }_{M} \bar{v}_{b}(a) \geq 1\right\}$ and define ${ }_{M} b_{s}=\vee\{a \in \mathcal{L}: a \in \hat{b}\}$. Otherwise, define ${ }_{M} b_{s}=I$. The mapping $b \rightarrow{ }_{M} b_{s}$ from $\mathcal{L} \rightarrow \mathcal{L}$ will be called the AC-operation on $\mathcal{L}$ relative to $M$. Note that when $\mathcal{M}$ is $\mathcal{L}$ and $M$ is $I$, we obtain the same AC-operation as defined in [4] for multiplicative lattices. It follows that ${ }_{M} v_{b} \leq{ }_{M} \bar{v}_{b}$. Also, ${ }_{M} \bar{v}_{b}$ is the smallest of all homogeneous pseudovaluations $\omega$ on $\mathcal{L}$ such that ${ }_{M} v_{b} \leq \omega$. Before continuing, we require the following results:

Theorem 1. Let $b$ be an element of $\mathcal{L}$ such that $b M \neq M$. Let a be an element in $\mathcal{L}$ and let $\alpha$ be a positive real number. A necessary and sufficient condition for ${ }_{M} \bar{v}_{b}(a) \geq \alpha$ is that for each pair of positive integers $p$ and $q$ such $0<p / q<\alpha$, there exists a positive integer $n$ such that $a^{q n} M \leq b^{p n} M$.

Proof. The proof of the theorem is largely a consequence of the definition of ${ }_{M} \bar{v}_{b}$. We leave the details of the proof to the reader.

As a consequence of Theorem 1, we note that $a \leq{ }_{M} b_{s}$ if and only if for each pair of positive integers $p$ and $q$ such that $0<p / q<1$, there is a positive integer $k$ such that $a^{q k} M \leq b^{p k} M$.

We now establish that the AC-operation an $\mathcal{L}$ relative to $M$ agrees with the same concept when specified to rings and modules. We see this by letting $R$ represent a commutative ring with identity and let $M$ be an Noetherian $R$-module. Let $b$ be an ideal in $R$ such that $b M \neq M$. A function $v_{b}^{M}: R \rightarrow \mathbb{Z}$ is defined the following way:

$$
v_{b}^{M}(x)=\left\{\begin{array}{l}
n, \text { if } x M \subseteq b^{n} M \text { but } x M \nsubseteq b^{n+1} M \\
\infty, \text { if } x M \subseteq b^{n} M, \text { for all positive integers } n
\end{array}\right.
$$

where for $n=0$, we have $b^{n}=R$. By defining $\bar{v}_{b}^{M}(x)=\lim _{n \rightarrow \infty} \frac{v_{b}^{M}\left(x^{n}\right)}{n}$, a homogeneous pseudovaluation is defined on $R$. The asymptotic closure of $b$ relative to $M$ is defined to be the set of all elements of $x \in R$ such that $\bar{v}_{b}^{M}(x) \geq 1$ [2]. Note that if $b M=M$, then the asymptotic closure of $b$ is $R$. If $\mathcal{L}$ is a lattice of ideals of $R$ then we see that the AC- operation on $\mathcal{L}$, relative to $M$, is exactly the asymptotic closure operation on $\mathcal{L}$, relative to $M$. Define $b_{y}^{M}=\left\{x \in R: \bar{v}_{b}^{M}(x) \geq 1\right\}$. Let $a \in \mathcal{L}$ such that $a \leq{ }_{M} b_{s}$. Then $M \bar{v}_{b}(a) \geq 1$. Assume that $p$ and $q$ are positive integers such that $0<p / q<1$. Choose a positive integer $k$ such that $a^{q k} M \leq b^{p k} M$. Thus, for each $x \in a$, we have $\bar{v}_{b}^{M}(x) \geq 1$. Therefore $a \subseteq b_{y}^{M}$ and ${ }_{M} b_{s} \leq b_{y}^{M}$.

Conversely, let $M$ be a Noetherian R-module, then the quotient ring $R /(0: M)$ is a commutative Noetherian ring which we denote as $\tilde{R}$ [10]. For each ideal $k$ of $R$, we let $\tilde{k}=k+(0: M) /(0: M)$ represent the corresponding ideal in $\tilde{R}$. The natural image in $\tilde{R}$ of an element $x$ in $R$ is represented by $\tilde{x}$. It follows that $M$ has a natural structure as an $\tilde{R}$-module. When $b_{y}^{M}$ is the asymptotic closure of $b$ in $R$ relative to $M$, then $\tilde{b}_{y}^{M}$ is the asymptotic closure of $\tilde{b}$ in $\tilde{R}$ [2, Lemma 1.5 , p.5]. Let $\tilde{\mathcal{L}}$ represent the multiplicative lattice of ideals in $\tilde{R}$. Since $\tilde{R}$ is an Noetherian ring, then the asymptotic closure of $\tilde{b}$ in 
$\tilde{\mathcal{L}}$ is also $\tilde{b}_{y}^{M}$ [4]. Thus for positive integers $p$ and $q$ such that $0<p / q<1$, pick a positive integer $k$ such that $\left(\tilde{b}_{y}^{M}\right)^{q k} \leq \tilde{b}^{p k}[4]$. Hence we have $\left(b_{y}^{M}\right)^{q k} M \leq b^{p k} M$ and $b_{y}^{M} \leq{ }_{M} b_{s}$ in $\mathcal{L}$.

As a consequence of the above discussion, we will henceforth refer to the AC-operation as the asymptotic closure operation.

The following six part property results mainly from the definitions of ${ }_{M} \bar{v}_{b}$ and ${ }_{M} b_{s}$, and is required for future reference.

Lemma 1. If $a$ and $b$ are elements in $\mathcal{L}$ such that $a M \neq M$ and $b M \neq M$, then:

1. $a M \leq b M$ implies ${ }_{M} \bar{v}_{a} \leq{ }_{M} \bar{v}_{b}$.

2. ${ }_{M} \bar{v}_{a} \leq{ }_{M} \bar{v}_{b}$ implies ${ }_{M} a_{s} \leq{ }_{M} b_{s}$.

3. $n_{M} \bar{v}_{a^{n}} \leq{ }_{M} \bar{v}_{a}$.

4. $\left({ }_{M} a_{s}\right)\left({ }_{M} b_{s}\right) \leq{ }_{M}(a b)_{s}$. Hence, $\left({ }_{M} b_{s}\right)^{n} \leq{ }_{M}\left(b^{n}\right)_{s}$.

5. $a \leq{ }_{M} b_{s}$ implies ${ }_{M} a_{s} \leq{ }_{M} b_{s}$.

6. ${ }_{M} a_{s} \leq{ }_{M} b_{s}$ implies ${ }_{M} \bar{v}_{a} \leq{ }_{M} \bar{v}_{b}$.

The Cancellation property: Here we show, by using means similar to Petro [7], that the cancellation property established for the asymptotic closure of elements of a multiplicative lattice having the ascending chain condition extends to the asymptotic closure of elements of a multiplicative lattice, relative to an $\mathcal{L}$-module having the ascending chain condition. First we recall the definition of a semi-prime operation on $\mathcal{L}$. A mapping $p: \mathcal{L} \rightarrow \mathcal{L}\left(a \rightarrow a_{p}\right)$ is a semi-prime operation on $\mathcal{L}$ if it satisfies the following conditions for all $a, b$ in $\mathcal{L}$ :

(a) $a \leq a_{p}$.

(b) $a \leq b$ implies $a_{p} \leq b_{p}$.

(c) $a_{p p}=a_{p}$.

(d) $a_{p} b_{p} \leq(a b)_{p}$.

We want to prove that the asymptotic closure operation on $\mathcal{L}$ relative to an $\mathcal{L}$-module (as defined above) is a semi-prime operation satisfying a cancellation law. We, therefore, review two necessary results:

Proposition 1. Let $M$ be the greatest element in $\mathcal{M}$ and let $a$ and $b \in \mathcal{L}$ such that $a M \neq M$ and $b M \neq M$. Then for all $c \in \mathcal{L}$, such that ${ }_{M} \bar{v}_{a b}(c) \neq 0$, we have

$$
\frac{1}{{ }_{M} \bar{v}_{a b}(c)} \leq \frac{1}{{ }_{M} \bar{v}_{a}(c)}+\frac{1}{{ }_{M} \bar{v}_{b}(c)}
$$


We also have the following result.

Proposition 2. Let $M$ be the greatest element of $\mathcal{M}$ such that $M \neq 0$. Let a and $b$ be elements of $\mathcal{L}$ such that $a M \neq M$ and $b M \neq M$. Then for all $c \in \mathcal{L}$, we have $\lim _{n \rightarrow \infty} n_{M} \bar{v}_{a^{n} b}(c)$ exists. Moreover, the following are also true:

(a) If there exists a positive integer $m$ such that $c^{m} M \leq b M$, then $\lim _{n \rightarrow \infty} n_{M} \bar{v}_{a^{n} b}(c)=$ ${ }_{M} \bar{v}_{a}(c)$.

(b) If for all positive integers $m$, we have $c^{m} M \leq b M$, then $\lim _{n \rightarrow \infty} n_{M} \bar{v}_{a^{n} b}(c)=0$.

(c) If there exists a positive integer $m$ such that $a^{m} M \leq b M$, then for all $c \in \mathcal{L}$, we have $\lim _{n \rightarrow \infty} n_{M} \bar{v}_{a^{n} b}(c)={ }_{M} \bar{v}_{a}(c)$.

The proofs of Propositions 1 and 2 follow using arguments similar to those given in [7] and we omit the details.

We now turn our attention to a cancellation law associated with the asymptotic closure of elements of $\mathcal{L}$, relative to $M$.

Theorem 2. (Cancellation Law) Let $a, b$, and $c$ be elements in $\mathcal{L}$, and let $M$ be the greatest element of $\mathcal{M}$. Then we have the following:

(a) If ${ }_{M}(a c)_{s} \leq{ }_{M}(b c)_{s}$ and if for some integer $m$, we have $a^{m} M \leq c M$, then ${ }_{M} a_{s} \leq$ ${ }_{M} b_{s}$.

(b) If ${ }_{M}(a c)_{s}={ }_{M}(b c)_{s}$ and if for some integer $m$, we have $(a \vee b)^{m} M \leq c M$, then ${ }_{M} a_{s}={ }_{M} b_{s}$.

By applying Lemma 1, we thus conclude that the asymptotic closure operation in $\mathcal{L}$ relative to $\mathrm{M}$ is a semi-prime operation. The remaining parts of the proof follow a pattern similar to [9].

In Theorem 1, we established that where $\alpha$ is a positive real number, we have ${ }_{M} \bar{v}_{b} \geq \alpha$ if and only if for each pair of positive integers $p$ and $q$ such that $0<p / q<\alpha$, there corresponds a positive integer $n$ such that $a^{q n} M \leq b^{p n} M$. We noticed that the integer $n$ was dependent on the rational number $p / q$. It can be shown that $n$ depends only on the element $b$ in $\mathcal{L}$ and this result gives a simpler characterization of the asymptotic closure relative to an element of a lattice module [cf. 4].

Lemma 2. Let $b$ be an element of $\mathcal{L}$ and let $M$ be the greatest element of $\mathcal{M}$. Then there exists a positive integer $n$ such that, for each pair of positive integers $p$ and $q$ such that $0<p / q<1$, we have $\left({ }_{M} b_{s}\right)^{n q} M \leq b^{n p} M$. Furthermore, $c \leq{ }_{M} b_{s}$ if and only if there exists positive integers $n$ and $k$ such that for all integers $i$, where $i \geq 1$, it is true that $\left(c^{n}\right)^{k+i} M \leq\left(b^{n}\right)^{i} M$.

Proof. For all integers $m \geq 1$, let $b_{m}=\left\{c \in \mathcal{L}: c^{m(k+1)} M \leq b^{m k} M\right.$ for all $\left.k \geq 1\right\}$ and let

$$
\left(b_{m}\right)^{\prime}=\{c \in \mathcal{L}: \text { for each integer } k \geq 1,
$$


there exists an integer $i \leq m$ such that we have $\left.c^{i(k+1)} M \leq b^{i k} M\right\}$.

Define $\beta_{m}=\left\{c M: c \in b_{m}\right\}$ and $\left(\beta_{m}\right)^{\prime}=\left\{c M: c \in\left(b_{m}\right)^{\prime}\right\}$. Then for each $m,\left(b_{m}\right)^{\prime} \subseteq$ $\left(b_{m+1}\right)^{\prime}$ and hence $\left(\beta_{m}\right)^{\prime} \subseteq\left(\beta_{m+1}\right)^{\prime}$. Since we know that $\mathcal{M}$ satisfies the ascending chain condition and $\left(\beta_{m}\right)^{\prime} \subseteq\left(\beta_{m+1}\right)^{\prime}$, pick an integer $n^{*}$ such that $\beta^{*}=\vee\left(\cup_{m=1}^{\infty}\left(\beta_{m}\right)^{\prime}\right)=$ $\vee\left(\beta_{n^{*}}\right)^{\prime}$. Since $b_{p} \subseteq b_{q}$ if $p$ divides $q$ then likewise $\beta_{p} \subseteq \beta_{q}$ whenever $p$ divides $q$. The definition of $b_{m}$ and $\left(b_{m}\right)^{\prime}$ imply that $b_{m} \subseteq\left(b_{m}\right)^{\prime}$. Consequently, $\left(\beta_{m}\right) \subseteq\left(\beta_{m}\right)^{\prime} \subseteq$ $\beta_{\Pi\{i: 0<i \leq m\}}$. It follows that $\beta^{*}=\vee \beta_{\Pi\left\{i: 0<i \leq n^{*}\right\}} \geq \vee \beta_{m}$ for all $m$. We then establish that there exists a positive integer $n$ such that $\beta^{*} \in \beta_{n}$. Next for each integer $k \geq 1$, we select a positive integer $h$ such that $\left({ }_{M} b_{s}\right)^{h(k+1)} M \leq b^{n k} M$. Then ${ }_{M} b_{s} M \leq \vee\left(\cup_{m=1}^{\infty}\left(\beta_{m}\right)^{\prime}\right)=\beta^{*}$. Since $\beta^{*} \in \beta_{n}$ for some integer $n$, it follows that ${ }_{M} b_{s} M \in \beta_{n}$ and hence for all positive integers $p$ and $q$ such that $0<p / q<1$, we have

$$
\left({ }_{M} b_{s}\right)^{n q} M \leq\left({ }_{M} b_{s}\right)^{n(p+1)} M \leq b^{n p} M .
$$

To prove the second part of the lemma, assume that $c \leq{ }_{M} b_{s}$. Pick a positive integer $n$ such that for each pair of positive integers $p$ and $q$ such that $0<p / q<1$, we have that $c^{n q} M \leq b^{n p} M$. In particular, for all integers $i \geq 1$ and $k \geq 1$, we see that $\left(c^{n}\right)^{k+i} M \leq\left(b^{n}\right)^{i} M$. Conversely, if we choose positive integers $n$ and $k$ such that for all $i \geq 1$, we have $\left(c^{n}\right)^{k+i} M<\left(b^{n}\right)^{i} M$, then

$$
\frac{{ }_{M} v_{b}\left(c^{n k+n i}\right)}{n(k+i)} \geq \frac{i}{k+i}
$$

Hence ${ }_{M} \bar{v}_{b}(c) \geq 1$ and $c \leq{ }_{M} b_{s}$.

We now turn our attention to a theorem that provides a simpler characterization of the asymptotic closure relative to an element of a lattice module.

Let b be an element of $\mathcal{L}$ and let ${ }_{M} b_{c}=\vee\{a \in \mathcal{L}$ : for all integers $i \geq 0$, there exists a positive integer $n$ such that $\left.a^{n+i} M \leq b^{i} M\right\}$. The element ${ }_{M} b_{c}$ is the asymptotic closure of $b$ relative to the element $M$ of a lattice module.

Theorem 3. If $b$ is an element in $\mathcal{L}$, then ${ }_{M} b_{c}={ }_{M} b_{s}$.

Proof. Let $W=\{c \in \mathcal{L}$ : there exists a positive integer $n$ such that for all $i \geq$ 0 , we have $\left.c^{n+i} M \leq b^{i} M\right\}$. If $e, d \in W$ then $d \vee e \in W$, hence ${ }_{M} b_{c} \in W$. Pick a positive integer $n$ such that for all $i \geq 0$, we have $\left({ }_{M} b_{c}\right)^{n+i} M<b^{i} M$. Thus, we have

$$
\frac{{ }_{M} v_{b}\left({ }_{M} b_{c}^{n+i}\right)}{(n+i)} \geq \frac{i}{n+i}
$$

and we see that $\lim _{n \rightarrow \infty} \frac{{ }_{M} v_{b}\left({ }_{M} b_{c}^{n+i}\right)}{(n+i)} \geq 1$ and ${ }_{M} b_{c} \leq{ }_{M} b_{s}$.

Next if $c \leq_{M} b_{s}$, choose a positive integer $n$, then for all $i \geq 0$, we have $\left(c^{n}\right)^{i+1} M \leq$ $b^{n i} M$. Also for each integer $i \geq 0$ there are integers $q$ and $r$ such that $i=n q+r$ where $0 \leq r<n$. Then we have, $c^{2 n+i} M=c^{2 n+n q+r} M \leq c^{2 n+n q} M \leq\left(b^{n}\right)^{q+1} M=b^{q n+n} M \leq$ $b^{q n+r} M=b^{i} M$. Therefore, ${ }_{M} b_{s} \leq{ }_{M} b_{c}$ and we have equality. 


\section{The Integral Closure Operation Relative to a Module}

In this section we develop the concept of the integral closure of an element of $\mathcal{L}$, a multiplicative lattice, relative to an element of $\mathcal{M}$, an $\mathcal{L}$-module with the ascending chain condition. The notions of the reduction and integral closure of an ideal in a commutative ring were introduced and studied by Northcott and Rees [6][8]. Lediaev [4] generalizes these operations in a Noether lattice setting. Sharp, Tiras, and Yassi [10] introduced similar operations in a ring, relative to a module. We obtain the same sort of results in this section. As we have done before, in this chapter we let $M$ represent the greatest element of the $\mathcal{L}$-module, $\mathcal{M}$.

First, we develop the concept of a-dependency of an element of $\mathcal{L}$ relative to $M$ and use this concept to establish the definition of a new mapping on $\mathcal{L}$.

Definition 1. An element $c$ in $\mathcal{L}$ is a-dependent on an element $b$ in $\mathcal{L}$ relative to $M$, if there exists a positive integer $n$ such that $c^{n+1} M \leq b(c \vee b)^{n} M$.

Let $b_{I}=\{m \in \mathcal{L}: m$ is a-dependent on $b$ relative to $M\}$; then define ${ }_{M} b_{a}=\vee b_{I}$.

The mapping $a: \mathcal{L} \rightarrow \mathcal{L}$ such that $b \rightarrow{ }_{m} b_{a}$ is called the IC-operation on $\mathcal{L}$ relative to $M$. The next lemma readily follows.

Lemma 3. Let $c$ and $b$ be elements of $\mathcal{L}$, then $c$ is a-dependent on $b$ relative to $M$ if and only if there exists a positive integer $n$ such that

$$
(b \vee c)^{n+1} M=b(b \vee c)^{n} M
$$

We now present other properties for the element ${ }_{M} b_{a}$ in $\mathcal{L}$. Clearly, if $c \in \mathcal{L}$ such that $c \leq b$, then $c$ is a-dependent on $b$ relative to $M$.

Lemma 4. If $c$ and $d$ are a-dependent on $b$ relative to $M$, then so is $c \vee d$. Therefore, we have ${ }_{M} b_{a}$ is a-dependent on $b$ relative to $M$.

Proof. Apply simple multiplicative lattice and lattice module properties along with the ascending chain condition on $\mathcal{M}$.

As was the case with the AC-operation, the IC-operation is also a semi-prime operation on $\mathcal{L}$.

Sharp, Tiras, Yassi [10] developed the concept of the integral closure of an ideal of a ring $R$ relative to a Noetherian $R$-module. They then established properties for this operation similar to the classical properties of the integral closure of an ideal. In this section, we will make some observations concerning the IC- operation in a lattice of ideals of a ring, relative to $M$, the greatest element of a lattice module.

In [2] we let $M$ be a Noetherian $R$-module and let $b$ be an ideal of $R$. An element $x$ of a ring $R$ is said to be integrally dependent on $b$ relative to $M$ if there exists a positive integer $n$ such that

$$
x^{n} M \subseteq \sum_{i=1}^{n} x^{n-i} b^{i} M .
$$


Moreover, when $M$ is a Noetherian R-module, then $R /(0: M)$ is a commutative Noetherian ring which we will denote as $\tilde{R}[10]$. For all ideals $k$ in $R$, we denote $\frac{k \vee(0: M)}{(0: M)}$ by $\tilde{k}$. Note that $M$ has a natural structure as an $\tilde{R}$-module.

We can now turn our attention to the following lemma found in [10]. Let $M$ be a Noetherian $R$-module. Denote the natural image in $\tilde{R}$ of an element $r$ in $R$ by $\tilde{r}$. Now let $x \in R$ and let $b$ be an ideal of $R$. It thus follows that $x$ is integrally-dependent on $b$ relative to $M$ if and only if $\tilde{x}$ is integrally-dependent on $\tilde{b}$ in $\tilde{R}$. Define $b_{a}^{M}=\{x \in R: x$ is integrally-dependent on $b$, relative to $M\}$. Hence, we have $b_{a}^{M}$ is the integral closure of $b$ relative to $M$, if and only if $\tilde{b}_{a}^{M}$ is the integral closure of $\tilde{b}$ in $\tilde{R}$.

The integral closure of an ideal relative to $M$ is also an ideal and we can show that if $\mathcal{L}$ is a lattice of ideals of a ring $R$ and $M$ is the greatest element of a lattice $\mathcal{L}$-module, then the IC-operation on $\mathcal{L}$ relative to $M$ is precisely the integral closure operation on $\mathcal{L}$ relative to $M$ as we show now. By [Lemma 4, Page 6], we know that in $\mathcal{L},{ }_{M} b_{a}$ is a-dependent on $b$ relative to $M$. Thus, select a postitve integer $k$ such that $\left({ }_{M} b_{a}\right)^{k+1} M=b\left({ }_{M} b_{a}\right)^{k} M$.

This last statement implies that $b$ and ${ }_{M} b_{a}$ have the same integral closure in $R$ relative to $M$. Let $b_{a}^{M}$ denote the integral closure of $b$ in $R$ relative to $M$. Hence, ${ }_{M} b_{a} \subset\left({ }_{M} b_{a}\right)_{a}^{M}=b_{a}^{M}$. Thus, ${ }^{M} b_{a} \subseteq b_{a}^{M}$. On the other hand, if we let $b_{a}^{M}$ be the integral closure of $b$ in $R$ relative to $M$, then $\tilde{b}_{a}^{M}$ represents the integral closure of $\tilde{b}$ in $\tilde{R}[10]$. Let $\tilde{\mathcal{L}}$ denote the lattice of ideals for $\tilde{R}$. Since $\tilde{R}$ is a Noetherian ring, the integral closure of $\tilde{b}$ in $\tilde{R}$ is equal to the integral closure of $\tilde{b}$ in $\tilde{\mathcal{L}}$ [4]. Hence, choose a positive integer $k$ such that $\left(\tilde{b}_{a}^{M}\right)^{k+1}=\tilde{b}\left(\tilde{b}_{a}^{M}\right)^{k}$ [4: Page 240, Lemma 7]. This implies $\left(b+b_{a}^{M}\right)^{k+1} M=b\left(b+b_{a}^{M}\right)^{k} M$. Hence, $b_{a}^{M} \subseteq M_{\tilde{R}} b_{a}$.

Next, let $c \subseteq{ }_{M} b_{a}=b_{a}^{M}$. Then $\tilde{c} \subseteq \tilde{b}_{a}^{M}$ in $\tilde{R}$ and $\tilde{\mathcal{L}}$. By [5, Theorem 2.43, Page 48], select a positive integer $k$ such that $\tilde{c}^{k+1} \subseteq b \widetilde{(b+c)}{ }^{k}$, therefore $c^{k+1} M \subseteq b(b+c)^{k} M$.

By applying the definition of ${ }_{M} b_{a}$, we find that if $c \in \mathcal{L}$ such that $c^{k+1} M \subseteq b(b+c)^{k} M$ then $c \subseteq M b_{a}$. As a consequence of the above, we will now call ${ }_{M} b_{a}$ the integral closure of $b$ relative to $M$. We also say that $b$ is integrally closed relative to $M$ if $b={ }_{M} b_{a}$.

\section{The Asymptotic and Integral Closure Operations in a Lattice Relative to an Element of a Lattice Module}

Throughout this section we will let $\mathcal{L}$ denote a multiplicative lattice and let $\mathcal{M}$ denote an $\mathcal{L}$-module with the ascending chain condition. We will show that where $M$, the greatest element of $\mathcal{M}$, is principal with $0: M=0$, and where $\mathcal{L}$ is modular, the asymptotic closure of an element in $\mathcal{L}$, relative to $M$, coincides with its integral closure, relative to $M$. This extends [Theorem 3 [4], (Page 241)], and also extends Nagata's similar result about rings [6]. We make use of the a-transform of $\mathcal{L}$ and a filtration of $\mathcal{M}$ to accomplish this.

First we review properties of these forms taken from [11].

A multiplicative filtration in $\mathcal{L}$ is a sequence $\left\{f_{i}\right\}$, where $i \in \mathbb{Z}$, of elements of $\mathcal{L}$ which satisfies the following: 
1. for all $i \leq 0$, we have $f_{i}=I$.

2. for all $i, k \in \mathbb{Z}$ we have $f_{i} f_{k} \leq f_{i+k}$.

Let $\left\{f_{i}\right\}$ be a fixed multiplicative filtration in $\mathcal{L}$ and consider the collection $R\left(\mathcal{L},\left\{f_{i}\right\}\right)$ of all formal sums $\sum_{i \in \mathbb{Z}} b_{i}$ of elements $b_{i}$ of $\mathcal{L}$ such that, for all $i \in \mathbb{Z}$ and for all $k \in \mathbb{Z}^{+}$ we have $f_{i} \geq b_{i} \geq b_{i+k}>f_{k} b_{i}$. Next, for elements $b=\sum_{i \in \mathbb{Z}} b_{i}$ and $c=\sum_{i \in \mathbb{Z}} c_{i}$ in $R\left(\mathcal{L},\left\{f_{i}\right\}\right)$, the following are defined:

1. $b \vee c=\sum_{i \in \mathbb{Z}}\left(b_{i} \vee c_{i}\right)$.

2. $b \wedge c=\sum_{i \in \mathbb{Z}}\left(b_{i} \wedge c_{i}\right)$.

3. $b c=\sum_{i \in \mathbb{Z}}\left(\bigvee_{r+t=i} b_{r} c_{t}\right)$.

4. $b \leq c$ if and only if, we have $b_{i} \leq c_{i}$, for all $i \in \mathbb{Z}$.

It also follows that for all integers $i$ and $k$, we have $b_{i+k} \geq f_{k} b_{i}$. The collection $R\left(\mathcal{L},\left\{f_{i}\right\}\right)$ together with the operations $\vee, \wedge, .$, and the relation $\leq$, forms a multiplicative lattice. Note that if $\mathcal{L}$ is modular, then $R\left(\mathcal{L},\left\{f_{i}\right\}\right)$ is also modular. Also for an element $b$ in $R\left(\mathcal{L},\left\{f_{i}\right\}\right)$ and an integer $i$, the $i$-th component of $b$ is represented $b_{i}$. It follows that $R\left(\mathcal{L},\left\{f_{i}\right\}\right)$ is a complete lattice [11].

If we let $a$ be an element of $\mathcal{L}$, where $a \neq I$ and if, for all $i \in \mathbb{Z}$, we let $f_{i}=a^{i}$, then the collection $R\left(\mathcal{L},\left\{a^{i}\right\}\right)$ is called the a-transform of $\mathcal{L}$ [3]. Observe that if $n$ is an integer such that $c \leq a^{n}$, then $c^{[n]}$ represents the least element, $d$, of $R\left(\mathcal{L},\left\{a^{i}\right\}\right)$ such that $d_{n} \geq c$.

Let $a$ be a fixed element in $\mathcal{L}$ such that $a \neq I$. We now summarize a number of results from [3] concerning a-transforms of $\mathcal{L}$. Let $n$ and $m$ be integers, and let $c$ and $d$ be elements of $\mathcal{L}$. Then, for the a-transforms $R\left(\mathcal{L},\left\{a^{i}\right\}\right)$ of $\mathcal{L}$, we have:

\section{Lemma 5.}

1. If $c \leq a^{n}$, then $c^{[n]}=\sum_{i \in \mathbb{Z}} c a^{i-n}$.

2. If $c \leq a^{n}$ and $d \leq a^{n}$, then $c^{[n]} \vee d^{[n]}=(c \vee d)^{[n]}$.

3. If $b \in R\left(\mathcal{L},\left\{a^{i}\right\}\right)$ and $c \leq a^{n}$ in $\mathcal{L}$ then $b c^{[n]}=\sum_{i \in \mathbb{Z}} b_{i-n} c$.

4. If $c$ and $d$ are elements of $\mathcal{L}$ such that $c \leq a^{n}$ and $d \leq a^{m}$ then $c^{[n]} d^{[m]}=(c d)^{[n+m]}$.

5. If $\mathcal{L}$ is modular and $b$ is a principal element of $\mathcal{L}$ such that $b \leq a^{n}$, then $b^{[n]}$ is principal in $R\left(\mathcal{L},\left\{a^{i}\right\}\right)$.

Next, we define filtrations in a lattice module. Let $\mathcal{M}$ be a lattice $\mathcal{L}$-module with greatest element $M$. A filtration in $\mathcal{M}$ is a sequence $\left\{F_{i}\right\}$ of elements of $M$, where $i$ is an integer, satisfying:

1. For all $i \leq 0$, we have $F_{i}=M$. 
2. For all integers $i$, we have $F_{i+1} \leq F_{i}$.

Consequently, it follows that for $b \in \mathcal{L}$, we have $\left\{b^{i} M\right\}$ is a filtration in $\mathcal{M}$. The elements of a multiplicative filtration in $\mathcal{L}$ are connected with elements of a filtration in $\mathcal{M}$ in the following way: Suppose $\left\{f_{i}\right\}$ is a multiplicative filtration in $\mathcal{L}$ and $\left\{F_{i}\right\}$ is a filtration in $\mathcal{M}$. Then, $\left\{F_{i}\right\}$ is an $\left\{f_{i}\right\}$ filtration in $\mathcal{M}$ if for all integers $i$ and $k$, we have $f_{k} F_{i} \leq F_{i+k}$.

Adopting the notation in [11], define $F\left(\mathcal{L}, \mathcal{M},\left\{f_{i}\right\},\left\{F_{i}\right\}\right)$ to be the set of all formal sums $\sum_{i \in \mathbb{Z}} B_{i}$ satisfying:

1. For all integers $i$, we have $B_{i} \in \mathcal{M}$.

2. For all non-negative integers $k$ and for all integers $i$, we have $f_{k} B_{i} \leq B_{i+k} \leq B_{i} \leq$ $F_{i}$.

We call $B_{i}$ the $i$-th component of $B=\sum_{i \in \mathbb{Z}} B_{i}$. For all $C \in F\left(\mathcal{L}, \mathcal{M},\left\{f_{i}\right\},\left\{F_{i}\right\}\right)$, we let $C_{i}$ denote the $i$-th component of $C$, so that $C=\sum_{i \in \mathbb{Z}} C i$. We will denote formal sums simply as $\sum B_{i}$ for elements in $F\left(\mathcal{L}, \mathcal{M},\left\{f_{i}\right\},\left\{F_{i}\right\}\right)$ and $\sum b_{i}$ for formal sums in $R\left(\mathcal{L},\left\{f_{i}\right\}\right)$.

For elements $B$ and $C$ in $F\left(\mathcal{L}, \mathcal{M},\left\{f_{i}\right\},\left\{F_{i}\right\}\right)$, where $B=\sum B_{i}$ and $C=\sum C_{i}$, the following definitions will be used: For all integers $i$, we say $B \leq C$ if and only if $B_{i} \leq C_{i}$. The set $F\left(\mathcal{L}, \mathcal{M},\left\{f_{i}\right\},\left\{F_{i}\right\}\right)$ forms a lattice under $\leq$ with the following meet and join properties:

- $B \wedge C=\sum_{i \in \mathbb{Z}}\left(B_{i} \wedge C_{i}\right)$;

- $B \vee C=\sum_{i \in \mathbb{Z}}\left(B_{i} \vee C_{i}\right)$;

The lattice will be represented by $R\left(\mathcal{L}, \mathcal{M},\left\{f_{i}\right\},\left\{F_{i}\right\}\right)[11]$. That $R\left(\mathcal{L}, \mathcal{M},\left\{f_{i}\right\},\left\{F_{i}\right\}\right)$ can be considered as an $R\left(\mathcal{L},\left\{f_{i}\right\}\right)$-module follows based on the following three properties that hold on elements in $R\left(\mathcal{L}, \mathcal{M},\left\{f_{i}\right\},\left\{F_{i}\right\}\right)$. Let $B \in R\left(\mathcal{L}, \mathcal{M},\left\{f_{i}\right\},\left\{F_{i}\right\}\right)$ :

(a) For all integers $i$ and $k$, we have $f_{k} B_{i} \leq B_{k+i}$.

(b) For all integers $i$, we have $\bigvee_{r \in \mathbb{Z}}\left(f_{r} B_{i-r}\right)=B_{i}$.

(c) Let $\sum b_{i} \in R\left(\mathcal{L},\left\{f_{i}\right\}\right)$ and $\sum B_{i} \in R\left(\mathcal{L}, \mathcal{M},\left\{f_{i}\right\},\left\{F_{i}\right\}\right)$. Then $\sum\left(\bigvee_{r \in \mathbb{Z}} b_{r} B_{i-r}\right)$ is an element of $R\left(\mathcal{L}, \mathcal{M},\left\{f_{i}\right\},\left\{F_{i}\right\}\right)$.

Define the following mapping $\mu[11], \mu: R\left(\mathcal{L}\left\{f_{i}\right\}\right) \times R\left(\mathcal{L}, \mathcal{M},\left\{f_{i}\right\},\left\{F_{i}\right\}\right) \rightarrow R(\mathcal{L}, \mathcal{M}$, $\left.\left\{f_{i}\right\},\left\{F_{i}\right\}\right)$ by the following equation:

$$
\mu(b, B)=\sum\left(\bigvee_{r \in \mathbb{Z}} b_{r} B_{i-r}\right) .
$$

We denote $\mu(b, B)$ as $b B$.

Let $b=\sum b_{i}$ be an element in $R\left(\mathcal{L},\left\{f_{i}\right\}\right)$ and let $B=\sum B_{i}$ be an element in $R\left(\mathcal{L}, \mathcal{M},\left\{f_{i}\right\},\left\{F_{i}\right\}\right)$. The lattice $R\left(\mathcal{L}, \mathcal{M},\left\{f_{i}\right\},\left\{F_{i}\right\}\right.$ is an $R\left(\mathcal{L}\left\{f_{i}\right\}\right)$-module, where $b B=$ $\left(\sum b_{i}\right)\left(\sum B_{i}\right)=\sum\left(\bigvee_{r \in \mathbb{Z}}\left(b_{r} B_{i-r}\right)\right)$. The following conclusions can be made: 
1. Where $b$ and $c \in R\left(\mathcal{L},\left\{f_{i}\right\}\right)$ and $D \in R\left(\mathcal{L}, \mathcal{M},\left\{f_{i}\right\},\left\{F_{i}\right\}\right)$, we have $(b c) D=b(c D)$.

2. For all $\alpha \in \Lambda$ let $b_{\alpha} \in R\left(\mathcal{L},\left\{f_{i}\right\}\right)$ and for all $\beta \in \Omega$, let $C_{\beta} \in R\left(\mathcal{L}, \mathcal{M},\left\{f_{i}\right\},\left\{F_{i}\right\}\right)$; then $\left(\vee b_{\alpha}\right)\left(\vee C_{\beta}\right)=\vee\left(b_{\alpha} C_{\beta}\right)$.

3. If $B \in R\left(\mathcal{L}, \mathcal{M},\left\{f_{i}\right\},\left\{F_{i}\right\}\right)$, then $\left(\sum f_{i}\right)(B)=B$.

4. $0 B=0$.

The next definition is important for proofs that follow:

Definition 2. Suppose $r$ is an integer and $C$ is an element in $\mathcal{M}$ with $C \leq F_{r}$. Define $C^{[r]}=\wedge\left\{B \in R\left(\mathcal{L}, \mathcal{M},\left\{f_{i}\right\},\left\{F_{i}\right\}\right): C \leq B_{r}\right\}$.

Note that $C^{[r]}=\sum_{r \in \mathbb{Z}}\left(f_{i-r} C\right)$.

Lemma 6 from [11] is needed for computations.

Lemma 6. Let $r$ be an integer and $b \in \mathcal{L}$ satisfying $b \leq f_{r}$. Let $s$ be an integer and let $C$ and $D$ be elements in $\mathcal{M}$ satisfying $C \leq F_{s}$, and $D \leq F_{s}$. Let $\sum a_{i} \in R\left(\mathcal{L}\left\{f_{i}\right\}\right)$ and $\sum E_{i} \in R\left(\mathcal{L}, \mathcal{M},\left\{f_{i}\right\},\left\{F_{i}\right\}\right)$. Then we have the following:

(a) $(C \vee D)^{[s]}=C^{[s]} \vee D^{[s]}$.

(b) $\left(\sum a_{i}\right) C^{[s]}=\sum\left(a_{i-s} C\right)$.

(c) $b^{[r]} C^{[s]}=(b C)^{[r+s]}$.

(d) Let $\mathcal{L}$ be a modular. If $r$ is an integer and $B$ is a principal element of $\mathcal{M}$ satisfying $B \leq F_{r}$, then $B^{[r]}$ is a principal element of $R\left(\mathcal{L}, \mathcal{M},\left\{f_{i}\right\},\left\{F_{i}\right\}\right)$.

Let $a^{\prime}$ and $s^{\prime}$ denote the integral closure and the asymptotic closure operations (relative to $\left.(I M)^{[0]}\right)$ in $R\left(\mathcal{L},\left\{a^{i}\right\}\right)$. We then have the following lemma:

Lemma 7. Let $\mathcal{L}$ be modular and let $b$ be an element of $\mathcal{L}$. Let $d$ be a principal element in $\mathcal{L}$ and let $M$ be a principal element in $\mathcal{M}$ with $0: M=0$. If $b \leq{ }_{M} d_{s}$, then $b$ is integrally-dependent on $d$ relative to $\mathcal{L}$. Therefore, we have ${ }_{M} d_{s}={ }_{M} d_{a}$.

Proof. Let $b \leq{ }_{M} d_{s}$, by Theorem 3, choose a positive integer $n$ such that for every non-negative integer $i$, we have

$$
b^{n+i} M \leq d^{i} M
$$

Hence, it follows that

$$
\begin{aligned}
& b^{n+i} M \leq\left(d^{i} M\right) \vee\left((d \vee b)^{n+i} M\right)=\left(d^{i} \wedge\left((d \vee b)^{n+i} M: M\right)\right) M \\
= & \left(d^{i} \wedge\left((d \vee b)^{n+i} \vee 0: M\right)\right) M=\left(d^{i} \wedge(d \vee b)^{n+i}\right) M=\left(d^{i}\left[(d \vee b)^{n+i}: d^{i}\right]\right) M .
\end{aligned}
$$

Since $\mathcal{M}$ has the ascending chain condition, select a positive integer $r$ such that, for all non-negative integers $i$, we have

$$
\left((d \vee b)^{n+r+i}: d^{r+i}\right) M=\left((d \vee b)^{n+r}: d^{r}\right) M
$$


Thus we say that

$\left.b^{n+r+1} M \leq d^{r+1}\left[(d \vee b)^{n+r+1}: d^{r+1}\right] M=d\left[\left(d^{r}(d \vee b)^{n+r}: d^{r}\right)\right] M\right) \leq d(d \vee b)^{n+r} M$

Therefore, $b$ is integrally-dependent on $d$ relative to $M$, and we conclude that ${ }_{M} d_{s}=$ ${ }_{M} d_{a}$.

Next we show that if $b \leq{ }_{M} a_{s}$, then $b^{[0]} \leq I_{s^{\prime}}^{[-1]}=I_{a^{\prime}}^{[-1]}$ in $R\left(\mathcal{L},\left\{a^{i}\right\}\right)$.

By Lemmas 5 part(e) and 6 part(d), we have $I^{[-1]}$ is a principal element in $R\left(\mathcal{L},\left\{a^{i}\right\}\right)$ and $(I M)^{[0]}$ is principal in $R\left(\mathcal{L}, \mathcal{M},\left\{a^{i}\right\},\left\{a^{i} M\right\}\right)$. Hence, by the first part of this theorem, we see that $I_{s^{\prime}}^{[-1]}=I_{a^{\prime}}^{[-1]}$. Since $b \leq{ }_{M} a_{s}$, choose an integer $n$ such that for all nonnegative integers $i, b^{n+i} M \leq a^{i} M$.

Consequently, for all non-negative integers $i$,

$$
\left(b^{n+i} M\right)^{[i]} \leq\left(a^{i} M\right)^{[i]} .
$$

Thus, we have

$$
\left(b^{n+i} M\right)^{[i]} \leq\left(a^{i}\right)^{[i]}(I M)^{[0]},
$$

and

$$
I^{[-i]}\left(b^{n+i} M\right)^{[i]} \leq I^{[-i]}\left(a^{i}\right)^{[i]}(I M)^{[0]} .
$$

Consequently,

$$
\left(b^{n+i} M\right)^{[0]} \leq I^{[-i]}(I M)^{[0]},
$$

and also

$$
\left(b^{n+i}\right)^{[0]}(I M)^{[0]} \leq I^{[-i]}(I M)^{[0]} .
$$

Therefore, it follows that

$$
\left(b^{[0]}\right)^{n+i}(I M)^{[0]} \leq\left(I^{[-1]}\right)^{i}(I M)^{[0]} .
$$

and

$$
b^{[0]} \leq I_{s^{\prime}}^{[-1]} .
$$

We conclude our work with the following theorem which shows that the asymptotic closure of an element of $\mathcal{L}$ relative to $M$ coincides with its integral closure relative to $M$ when $\mathcal{L}$ is modular and $0: M=0$. This theorem extents the lattice theoretic results of Lediaev [4] and the Nagata's result that the asymptotic and integral closures coincide in Noetherian rings [6].

Theorem 4. Let $\mathcal{L}$ be modular and let $M$ be a principal element of $\mathcal{M}$ with $0: M=0$. For all elements a in $\mathcal{L}$, we have ${ }_{M} a_{a}={ }_{M} a_{s}$. Furthermore, $b \leq_{M} a_{s}$ implies that $b$ is integrally-dependent on a, relative to $M$.

Proof. Let $b$ and $a$ be elements in $\mathcal{L}$. Let $b \leq{ }_{M} a_{s}$ and $(I M)^{[0]}$ be an element in $R\left(\mathcal{L}, \mathcal{M},\left\{a^{i}\right\},\left\{a^{i} M\right\}\right)$. Thus, by Lemma 7 , we know that in $R\left(\mathcal{L},\left\{a^{i}\right\}\right)$, we have 
$b^{[0]} \leq I_{a^{\prime}}^{[-1]}$, and we see that $b^{[0]}$ is integrally-dependent on $I^{[-1]}$ relative to $(I M)^{[0]}$. Choose a positive integer $n$ such that

$$
\left(I^{[-1]} \vee b^{[0]}\right)^{n+1}(I M)^{[0]}=I^{[-1]}\left(I^{[-1]} \vee b^{[0]}\right)^{n}(I M)^{[0]} .
$$

The left side of (1) is equal to

$$
\begin{aligned}
& \bigvee_{i=0}^{n+1}\left(b^{[0]}\right)^{i}\left(I^{[-1]}\right)^{n+1-i}(I M)^{[0]}=\left(\bigvee_{i=0}^{n+1}\left(b^{[0]}\right)^{i}\left(I^{[-1]}\right)^{n+1-i}\right)(I M)^{[0]} \\
= & \left(I^{[-n-1]} \vee \cdots \vee\left(b^{i}\right)^{[i-1-n]} \vee \cdots \vee\left(b^{n+1}\right)^{[0]}\right)(I M)^{[0]}
\end{aligned}
$$

The zero component of this part is $\left(\bigvee_{i=0}^{n+1} b^{i} a^{n+1-i} M\right)=(a \vee b)^{n+1} M$.

The right side of (1) is equal to

$$
\begin{aligned}
& I^{[-1]}\left(\bigvee_{i=0}^{n}\left(b^{[0]}\right)^{i}\left(I^{[-1]}\right)^{n-i}\right)(I M)^{[0]} \\
= & \left(I^{[-n-1]} \vee \cdots \vee\left(b^{n-i}\right)^{[-i-1]} \vee \cdots \vee\left(b^{n}\right)^{[-1]}\right)(I M)^{[0]} .
\end{aligned}
$$

The zero component of this side is $\left(\bigvee_{i=0}^{n} b^{i} a^{n+1-i} M\right)=a(a \vee b)^{n} M$.

Therefore, $(a \vee b)^{n+1} M=a(a \vee b)^{n} M$, and $b$ is integrally-dependent on $a$ relative to $M$.

\section{References}

[1] R. P. Dilworth, Abstract commutative ideal theory, Pacific Journal of Mathematics 12 (1962), 481-498.

[2] S. Foster and J. A. Johnson, The asymptotic closure of an ideal relative to a module, Tamkang Journal of Mathematics 32 (2001), 231-236.

[3] E. W. Johnson, A -transforms and Hilbert functions in local lattices, Trans. Amer. Math. Soc. 137 (1969), 125-139.

[4] J. Lediaev, Asymptotic and integral closure of the elements in multiplicative lattices, Michigan Math. J. 16(1969), 235-243.

[5] J. Lediaev, Semi-Prime Operations on Noether Lattices, Thesis (Riverside, California), 1967.

[6] M. Nagata, Note on a paper of Samuel concerning asymptotic properties of the ideals, Mem. Coll. Sci. Univ. Kyoto Ser. A Math. 30 (1957), 166-175.

[7] J. W. Petro, Some results on the asymptotic completion of an ideal, Pro. Amer.Math.Soc. 15(1964), 519-524.

[8] D. Rees, Valuations associated with a local ring, I. Proc. London Math. Soc. 5 (1955), 107-128.

[9] P. Samuel, Some asymptotic properties of powers of ideals, Ann. of Math. 56 (1952), 11-21.

[10] R. Y. Sharp, Y. Tiras and M. Yassi, Integral closure of ideals, J. London Math. Soc. 42(1990), 385-392.

[11] M. Taylor, Dissertation, University of Houston, 1988. 
Department of Mathematics, University of Houston, Houston, Texas 77204-3008, USA.

Department of Mathematics, University of Houston, Houston, Texas 77204-3008,USA.

E-mail: jjohnson@math.uh.edu 